Published in "Hyperfine Interactions doi:10.1007/s10751-011-0386-5, 2011"

which should be cited to refer to this work.

\title{
The size of the proton
}

\section{From the Lamb-shift in muonic hydrogen}

T. Nebel - A. Antognini - F. D. Amaro • F. Biraben - J. M. R. Cardoso • D. S. Covita • A. Dax · S. Dhawan - L. M. P. Fernandes · A. Giesen · T. Graf • T. W. Hänsch • P. Indelicato • L. Julien • C.-Y. Kao • P. Knowles • F. Kottmann • E. Le Bigot • Y.-W. Liu • J. A. M. Lopes · L. Ludhova • C. M. B. Monteiro • F. Mulhauser • F. Nez • P. Rabinowitz • J. M. F. dos Santos • L. A. Schaller • K. Schuhmann • C. Schwob · D. Taqqu • J. F. C. A. Veloso • R. Pohl

\author{
Abstract The root-mean-square (rms) charge radius $r_{\mathrm{p}}$ of the proton has so far \\ been known only with a surprisingly low precision of about $1 \%$ from both electron \\ scattering and precision spectroscopy of hydrogen. We have recently determined $r_{\mathrm{p}}$ \\ by means of laser spectroscopy of the Lamb shift in the exotic "muonic hydrogen" \\ T. Nebel $(\bowtie) \cdot$ A. Antognini $\cdot$ T. W. Hänsch $\cdot$ R. Pohl \\ Max-Planck-Institut für Quantenoptik, Garching, Germany \\ e-mail: tbn@mpq.mpg.de \\ F. D. Amaro - J. M. R. Cardoso - L. M. P. Fernandes · J. A. M. Lopes · C. M. B. Monteiro \\ J. M. F. dos Santos \\ Departamento de Física, Universidade de Coimbra, Coimbra, Portugal \\ F. Biraben · P. Indelicato · L. Julien · E. Le Bigot · F. Nez · C. Schwob \\ Laboratoire Kastler Brossel, Ecole Normale Supérieure, CNRS, Paris, France \\ F. Biraben · P. Indelicato $\cdot$ L. Julien $\cdot$ E. Le Bigot $\cdot$ F. Nez $\cdot$ C. Schwob \\ Université P. et M. Curie, Paris, France \\ D. S. Covita · J. F. C. A. Veloso \\ I3N, Departamento de Física, Universidade de Aveiro, Aveiro, Portugal \\ A. Dax · S. Dhawan \\ Physics Department, Yale University, New Haven, CN, USA \\ A. Giesen $\cdot$ K. Schuhmann \\ Dausinger \& Giesen GmbH, Stuttgart, Germany \\ T. Graf \\ Institut für Strahlwerkzeuge, Universität Stuttgart, Stuttgart, Germany \\ T. W. Hänsch \\ Ludwig-Maximilians-Universität, Munich, Germany \\ C.-Y. Kao · Y.-W. Liu \\ Physics Department, National Tsing Hua University, Hsinchu, Taiwan
}


atom. Here, the muon, which is the 200 times heavier cousin of the electron, orbits the proton with a 200 times smaller Bohr radius. This enhances the sensitivity to the proton's finite size tremendously. Our new value $r_{\mathrm{p}}=0.84184$ (67) fm is ten times more precise than the generally accepted CODATA-value, but it differs by 5 standard deviations from it. A lively discussion about possible solutions to the "proton size puzzle" has started. Our measurement, together with precise measurements of the $1 \mathrm{~S}-2 \mathrm{~S}$ transition in regular hydrogen and deuterium, also yields improved values of the Rydberg constant, $R_{\infty}=10,973,731.568160(16) \mathrm{m}^{-1}$.

Keywords Muonic hydrogen $\cdot$ Lamb shift $\cdot$ Laser spectroscopy $\cdot$ Proton charge radius

\section{Introduction}

Over the last decades, simple atomic systems, mostly a compound of one negatively and one positively charged particle, have served as vastly successful probes of the basic aspects of the laws of physics. It was the extensive investigation of the hydrogen atom in the early part of the 20th century that led to the birth of modern quantum mechanics when Heisenberg reinterpreted the classical picture of kinematics and mechanics in a quantum theoretical way in 1925 [1]. Three years later in 1928, Dirac unified the concepts of relativity and quantum mechanics [2]. The discovery of the Lamb shift in atomic hydrogen [3] in 1947 motivated the description of physical processes via quantum field theories. Since then, many hydrogen-like systems have been considered both experimentally and theoretically, providing a fertile soil for refining our abstract portraiture of Nature's laws.

A vivid example for this development is the confirmation of quantum electrodynamics (QED) of bound systems. Prominent QED-tests are manifold, among them the investigations on exotic systems such as muonium [4] or positronium [5]. Among the most accurate test-instruments, however, are the absolute frequency measurements of (laser induced) transitions in simple atomic systems. Here, the Doppler-free two-photon spectroscopy of atomic hydrogen $[6,7]$ serves as the most prosperous instance of the confirmation of fundamental physics. The $1 \mathrm{~S}-2 \mathrm{~S}$ transition frequency

P. Knowles $\cdot$ L. Ludhova $\cdot$ F. Mulhauser $\cdot$ L. A. Schaller

Département de Physique, Université de Fribourg, Fribourg, Switzerland

F. Kottmann

Institut für Teilchenphysik, ETH Zürich, Zürich, Switzerland

P. Rabinowitz

Department of Chemistry, Princeton University, Princeton, NJ, USA

D. Taqqu

Paul Scherrer Institut-PSI, 5232 Villigen, Switzerland

Present Address:

A. Antognini

ETH Zürich, Zürich, Switzerland 
was measured with a relative uncertainty of $1.4 \cdot 10^{-14}$ [8]. However, bound-state QED tests were limited to a precision level of a few parts per million. This stems from the fact that a central constant employed in the theoretical QED-calculations, the root mean square (rms) proton charge radius $r_{p}$, is independently only known to $1-2 \%$ accuracy $[9,10]$. To overcome this limitation in testing bound-state QED, our experiment intends to provide a 20 times more accurate independent value for $r_{p}$ by measuring the $2 \mathrm{~S}-2 \mathrm{P}$ Lamb shift in muonic hydrogen ( $\mu \mathrm{p})$.

In this article, the experiment to measure the Lamb shift in $\mu p$ at a proton accelerator facility and the method to extract the $2 \mathrm{~S}-2 \mathrm{P}$ resonance line position are described.

\section{History of the experiment}

Following the measurements of the classical Lamb shift [3] in 1947 and of the magnetic moment of the electron in 1948 [11], bound state QED started developing. Looking for possible test grounds for the vacuum polarization terms of QED, Vernon Hughes (Yale University, USA), Val L. Teledgi (University of Chicago, USA), and Emilio Zavattini (CERN) considered the measurement of the 2S-2P energy difference in $\mu p$ in the late 1960s [12]. In 1969, the theoretician A. DiGiacomo (CERN) provided the first accurate QED-calculation of the 2S-2P energy difference in $\mu \mathrm{p}$ [13].

The first $2 \mathrm{~S}$-lifetime measurements in $\mu$ p were proposed at Columbia University (USA) already in 1971, but the crucial existence of the so-called long-lived 2S component could only be verified by our group three decades later [14, 15]. In the 1980s, the aim of the Lamb shift experiment changed from testing vacuum polarization terms towards the determination of the proton charge radius. This was motivated by the tremendous improvement in H-spectroscopy (in particular 1S-2S), which called for a more accurate and independent value of the proton charge radius, required to compare experiment to QED theory.

With a new low-energy muon beamline and a new concept for the $6 \mu \mathrm{m}$ laser, the Lamb shift in $\mu$ p was proposed to be measured at the PSI proton accelerator in 1998. A first engineering-run with the complete system working was performed by our collaboration in 2002. The first data-taking run in 2003 as well as the second run scheduled only for 2007 were successful but did not yield a resonance line. Before the last run in 2009, we developed a new thin disk pump laser replacing the much too slow eximer-dye pump lasers. We also improved the data acquisition electronics, data analysis tools, and many important details of the apparatus. During the successful, four-month-long beamtime in 2009, we finally discovered the much sought-after resonance line and experimentally determined the Lamb shift in $\mu$ p for the first time. Data for three more transitions in $\mu \mathrm{p}$ and $\mu \mathrm{d}$ was recorded and the analysis is currently underway.

\section{Experiment}

We have measured Lamb shift in muonic hydrogen ( $\mu$ p), a two-body compound of a proton and a negatively charged muon [16]. The transition presented here is 
between the $2 \mathrm{~S}_{1 / 2}^{F=1}-2 \mathrm{P}_{3 / 2}^{F=2}$ atomic levels. The Lamb shift experiment is conducted at the proton accelerator of the Paul Scherrer Institute (PSI) in Switzerland. ${ }^{1}$ Here, a proton beam is accelerated to $2 \mathrm{~mA}$ at $590 \mathrm{MeV}$ and collides with a $40 \mathrm{~mm}$ thick rotating-wheel $(1 \mathrm{~Hz})$ carbon target. Our experiment makes use of the secondary particles emitted through the $\pi$ E5 port [17], a low momentum (10-120 MeV/c) pion and muon beam line looking at the target at an angle of $175^{\circ}$ with respect to the proton beam. This is by far the most intense low-energy meson beam in the world.

The principle of the experiment is to stop low-energy muons in dilute hydrogen gas, form muonic hydrogen in the $2 \mathrm{~S}$ state and, delayed with respect to the formation time of the muonic atom, drive the $2 \mathrm{~S}-2 \mathrm{P}$ atomic transition by means of an infra-red laser at $6 \mu \mathrm{m}$. The emission of $1.9 \mathrm{keV} \mathrm{K} \mathrm{K}_{\alpha} \mathrm{X}$-rays during the instantaneous decay of the $2 \mathrm{P}$ state to the ground state serves as a signature for the induced transition.

Slow negative muons are decelerated in low-pressure $(1.0 \mathrm{hPa})$ hydrogen $\left(\mathrm{H}_{2}\right)$ gas before they are captured and $\mu^{-} \mathrm{p}$ atoms in highly excited states $(n \approx 14)$ are formed [18-20]. At this principle quantum number, the overlap of muonic and electronic wave functions is maximal and $n$ can be estimated from $n \approx \sqrt{m_{r}^{\mu \mathrm{p}} / m_{r}^{\mathrm{ep}}} \approx 14$, with $m_{r}^{\mu \mathrm{p}}$ and $m_{r}^{\mathrm{ep}}$ being the reduced masses of the $\mu \mathrm{p}$ and the $\mathrm{H}$ atom. Through a series of different de-excitation processes (see, e.g. $[18,21]$ ), most of the $\mu^{-} p$ atoms quickly cascade down to the $1 \mathrm{~S}$ ground state, thereby emitting a prompt $\mathrm{K}$-line $\mathrm{X}$ ray. For $0.6 \mathrm{hPa}$, the cascade time has been determined to be $T_{\text {cas }}^{\mathrm{\mu p}}=(37 \pm 5) \mathrm{ns}$ [22]. At $1.0 \mathrm{hPa}$, only a fraction $\epsilon_{2 \mathrm{~S}}=(2.76 \pm 0.17) \%$ of the $\mu \mathrm{p}$ atoms reaches the $2 \mathrm{~S}$ state [23-25]. Only $1.1 \pm 0.1 \%$ of the total number of $\mu$ p atoms form long-lived $\mu \mathrm{p}(2 \mathrm{~S})$ with a lifetime $\tau_{2 \mathrm{~S}}^{\text {long }}=1.04_{-0.21}^{+0.29} \mu \mathrm{s}[15,26]$.

Triggered by an incoming muon, a short laser pulse with a wavelength tunable around $\lambda \approx 6 \mu \mathrm{m}$ (corresponding to the $2 \mathrm{~S}-2 \mathrm{P}$ energy difference) fills a mirror cavity surrounding the muon stop volume, about $0.9 \mu$ s after the muon stop and the cascade. When the laser is on resonance, $\mu \mathrm{p}$ atoms in the $2 \mathrm{~S}$ state can be excited to the $2 \mathrm{P}$ state from where they decay to the $1 \mathrm{~S}$ ground state instantaneously (lifetime $\tau_{2 \mathrm{P}}=8.6 \mathrm{ps}$ ). The emitted $1.9 \mathrm{keV} \mathrm{K}_{\alpha} \mathrm{X}$-rays, which occur in time-coincidence with the laser pulse (termed delayed $\mathrm{K}_{\alpha} \mathrm{X}$-rays), serve as a signal and are recorded at different laser wavelengths. A resonance curve is obtained by plotting the number of delayed X-rays (normalized to the prompt X-rays to account for beam-intensity variations) versus the laser wavelength.

To efficiently decelerate muons within the muon lifetime of $2.2 \mu$ s and, at the same time, to reduce the background drastically is the aim of a newly developed low energy muon beam [27]. It consists of the cyclotron trap [28-30] acting as a magnetic bottle confining and decelerating the muons, a muon extraction channel acting as a momentum filter guiding and separating the low-energy muons from unwanted background, and a 5 Tesla superconducting solenoid housing the muon detectors and the hydrogen target. A schematic layout is shown in Fig. 1.

Upon entering the hydrogen target, the muons pass two muon detectors consisting of stacks $S_{1}$ and $S_{2}$ of ultra-thin carbon foils $\left(d=4 \mu \mathrm{g} / \mathrm{cm}^{2}\right)$ and a velocity filter (Fig. 2). The whole arrangement acts as a non-destructive transmission detector for $\mathrm{keV}$-muons, reduces the muon energy and frictionally cools the muon beam. Passing the carbon foils, the muons extract secondary electrons which are separated from

$\overline{{ }^{1} \text { Paul Scherrer Institute, } 5232}$ Villigen PSI, Switzerland, http://www.psi.ch. 


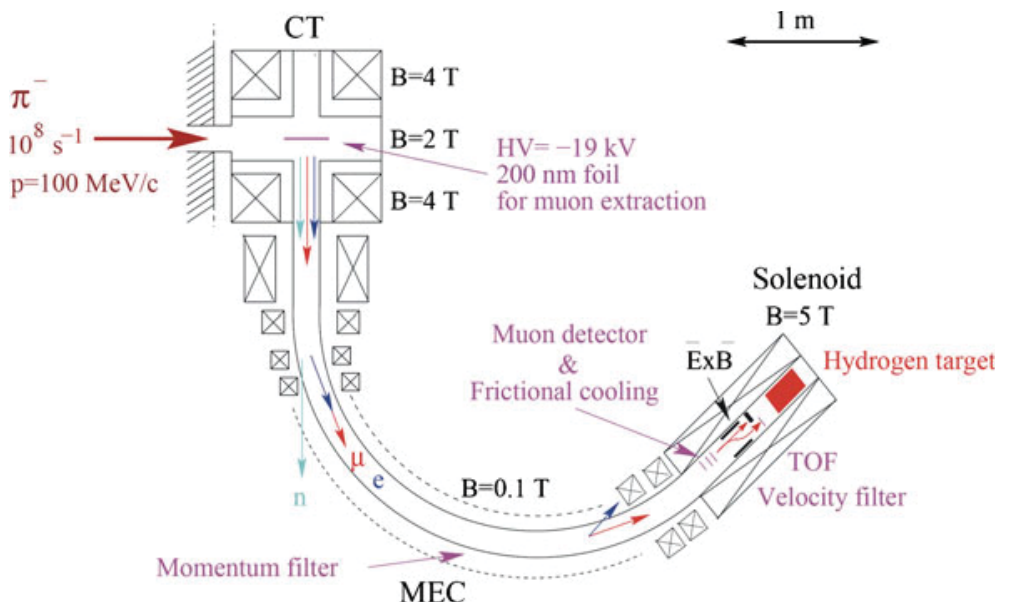

Fig. 1 Low-energy muon beam line. A schematic layout of the low-energy muon beam in the $\pi \mathrm{E} 5$ area at PSI. Shown are the cyclotron trap (CT), the muon extraction channel (MEC) and the target solenoid

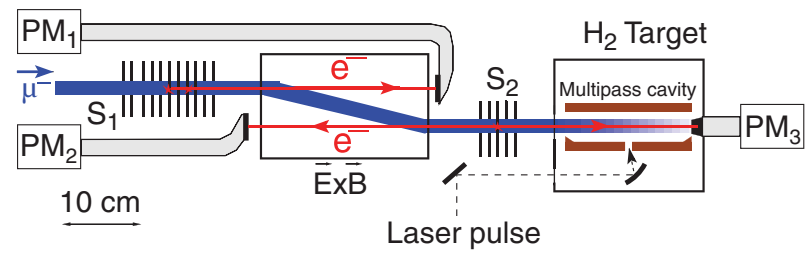

Fig. 2 Schematic of the target and muon detectors. Muons entering from the left have to pass two stacks of carbon foils as well as an $\mathbf{E} \times \mathbf{B}$ velocity filter. Secondary electrons are detected in plastic scintillators monitored by photo multiplier tubes. The gas-target is filled with dilute $\mathrm{H}_{2}$-gas and houses the target mirror cavity. Two arrays of LAAPDs are mounted on top and below the gasvolume (not shown)

the relatively slow muons when passing through the $\mathbf{E} \times \mathbf{B}$ velocity filter. Electrons emitted from $S_{1}$ and $S_{2}$ are detected in coincidence yielding a very clean nondestructing muon-detection signal supplying an electrical trigger for the laser system. The muons loose about $2.5 \mathrm{keV}$ of kinetic energy while passing through each of the five carbon foils and release secondary electrons. The electric potentials for the foils are chosen such that the energy loss is partly compensated before hitting the next carbon foil $(\Delta U=1.8 \mathrm{kV})$, thereby cooling the muons frictionally. Above and below the muon beam, two face-to-face rows of 10 large area avalanche photo-diodes (LAAPDs, $14 \times 14 \mathrm{~mm}^{2}$ active area) are mounted together with their pre-amplifiers and record the $1.9 \mathrm{keV} \mathrm{K}_{\alpha} \mathrm{X}$-rays in a distance of $8 \mathrm{~mm}$ from the muon beam axis.

The laser system employed for our experiment is sketched in Fig. 3 [31, 32]. Two ytterbium-YAG (Yb:YAG) thin-disk laser units pump the entire pulsed laser system and provide $53 \mathrm{~mJ}$ of green laser pulses at $515 \mathrm{~nm}$ after second harmonic generation (SHG). Their pulses have a delay of only 400 ns relative to the muon trigger signal. They pump an injection seeded master oscillator power amplifier (MOPA) titanium sapphire (Ti:Sapph) laser system which delivers $15 \mathrm{~mJ}$ red pulses around $\lambda \approx 708 \mathrm{~nm}$. 
Fig. 3 Schematic of the laser system. Infra-red laser pulses are obtained by sending strong $708 \mathrm{~nm}$ laser pulses through a $\mathrm{H}_{2}$ filled Raman shifter. The red pulses are produced in a Ti:Sapph MOPA subsystem which is pumped by a

frequency doubled thin-disk laser unit and injection seeded by a wavelength controlled and tunable cw Ti:Sapph laser. The depicted abbreviated components are: Fabry-Pérot reference cavity (FP), iodine and cesium absorption cells $\left(\mathrm{I}_{2} / \mathrm{Cs}\right)$, second harmonic generation (SHG), water absorption cell $\left(\mathrm{H}_{2} \mathrm{O}\right)$, and fast infra-red photo diodes to monitor the light going into and leaking out of the target cavity

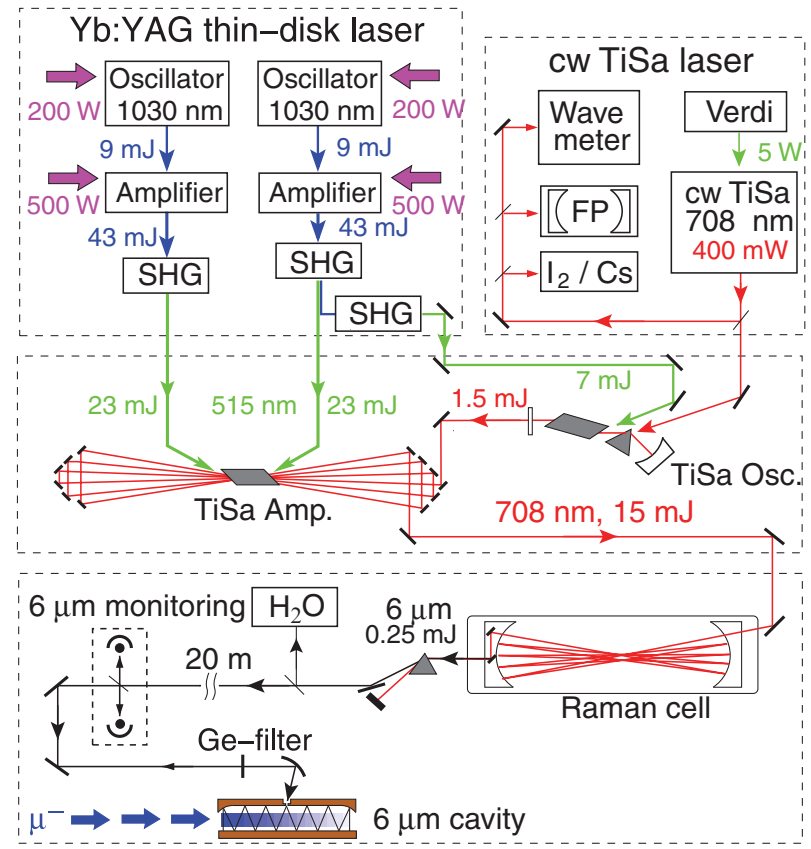

At the output of the Ti:Sapph amplifier, the laser light enters an efficient and reliable $3^{\text {rd }}$-Stokes Raman shifter [33] (termed Raman cell, RC) operated at 15.5 bar of $\mathrm{H}_{2}$ gas. Here, its wavelength is converted to the infra-red domain $(\lambda=5.48$ $6.04 \mu \mathrm{m})$. The RC introduces a constant Raman shift $\left(\Delta \widetilde{v}=3 \times 4,155.2 \mathrm{~cm}^{-1}\right)$ so that the frequency tuning of the $\mathrm{cw}$ Ti:Sapph laser translates directly into the infrared. In such a way, the frequency control of the $6 \mu \mathrm{m}$ laser can be performed directly in the much more convenient visible region. The frequency control of the Ti:Sapph oscillator is managed by seeding it with a single-mode continuous wave (cw) Ti:Sapph laser. The absolute frequency calibration of the $\mathrm{cw}$ Ti:Sapph subsystem is achieved by a wavemeter and by iodine and cesium absorption cells. Water absorption spectroscopy is utilized to accurately calibrate the whole laser system directly in the infra-red domain yielding the laser frequency calibration to be accurate to $300 \mathrm{MHz}$.

\section{Results}

With the experimental equipment described in the last section, a measurement run with 8 weeks of setup time and a data aquisition time of 15 weeks was successfully completed in August 2009. Figure 4 shows the time spectra of the $1.9 \mathrm{keV}$ X-rays split into two groups, one on-resonance and one off-resonance. The on-resonance time spectrum is produced from events recorded $\pm 17 \mathrm{GHz}$ around the resonance peak, which has a natural linewidth of $18.6 \mathrm{GHz}$. Events from outside this range contribute to the time spectrum of the off-resonance data. In the plots, the prompt peak caused by the muonic cascade during $\mu$ p formation is indicated in blue. For each 


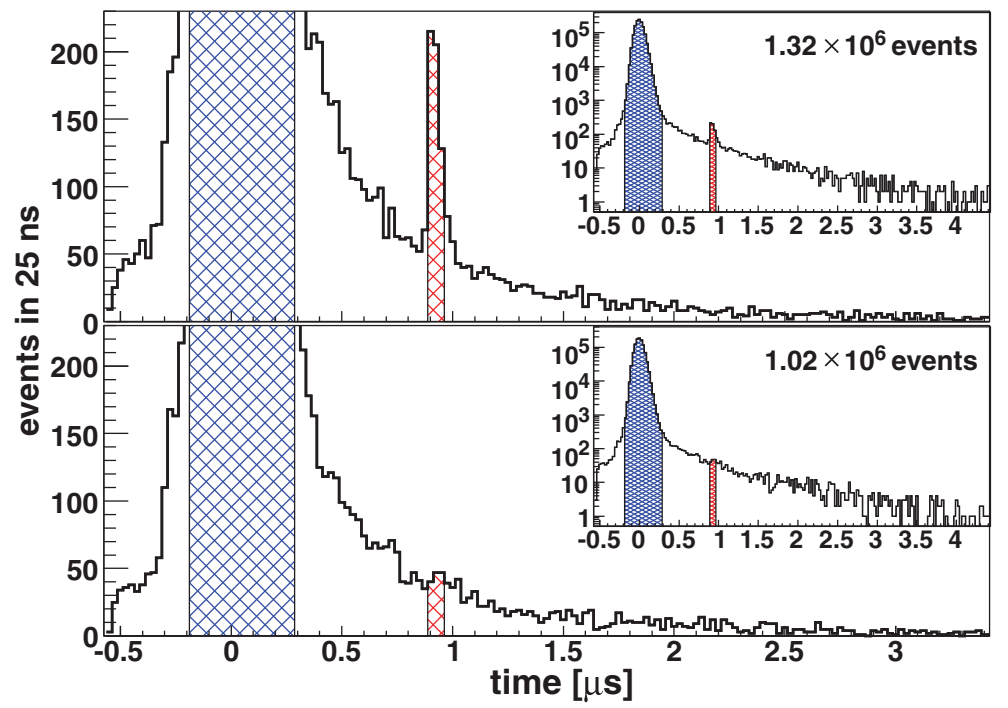

Fig. 4 Accumulated time spectra on and off resonance. Summed X-ray time spectra recorded on resonance (top) and off resonance (bottom) shown in linear and logarithmic scale. The LTW $t \in[0.887,0.962] \mu \mathrm{s}$ is indicated as well as the contribution of the prompt X-rays

laser frequency, it serves to normalize the data to the number of formed $\mu \mathrm{p}$ atoms. The $75 \mathrm{~ns}$ wide laser time window (LTW, $t \in[0.887,0.962] \mu \mathrm{s}$ ) is marked in red.

The $2 \mathrm{~S}-2 \mathrm{P}$ resonance line is plotted in Fig. 5. It is obtained by plotting the number of $\mathrm{K}_{\alpha}$ events recorded in the LTW, normalized to the number of prompt events, as a function of the laser frequency. The fit to the data is a simple Lorentzian profile on top of a flat background. We have in total measured 550 events in the resonance where 155 background events are expected. All four parameters, namely the position of the Lorentzian, its amplitude, and its width as well as the background amplitude are varied freely.

A centroid position of $49,881.88$ (70) $\mathrm{GHz}$ and a width of 18.0 (2.2) $\mathrm{GHz}$ were obtained. The given uncertainties are the statistical $1 \sigma$ confidence intervals. The width agrees well with the width of 20 (1) GHz expected from a Doppler- and powerbroadened natural line-width of $18.6 \mathrm{GHz}$ convoluted with the laser bandwidth. The fit to the data shows a $\chi^{2}=28.1$ for 28 degrees of freedom (dof). The systematic uncertainty of $300 \mathrm{MHz}$ is exclusively dominated by the laser frequency calibration. Other sources of systematic corrections like Zeeman shift in the $5 \mathrm{~T}$ field, Stark shifts, Doppler shift, pressure shift, and recoil shift are well below $30 \mathrm{MHz}$ and do not contribute noticeably. Adding the uncertainties in quadrature, one obtains a centroid position for the $2 \mathrm{~S}_{1 / 2}^{F=1}-2 \mathrm{P}_{3 / 2}^{F=2}$ transition of $49,881.88$ (76) $\mathrm{GHz}$ corresponding to an energy-difference of $\Delta E=206.2949$ (32) meV. With the current status of the $\mu \mathrm{p}$-theory [35], we deduce a rms proton charge radius $r_{\mathrm{p}}=0.84184$ (36)(56) fm, where the first uncertainty corresponds to our experimental uncertainty of $760 \mathrm{MHz}$. The second uncertainty stems from the theoretical uncertainty mainly due to proton polarizability terms and dominates our total relative uncertainty of $8 \times 10^{-4}$. 


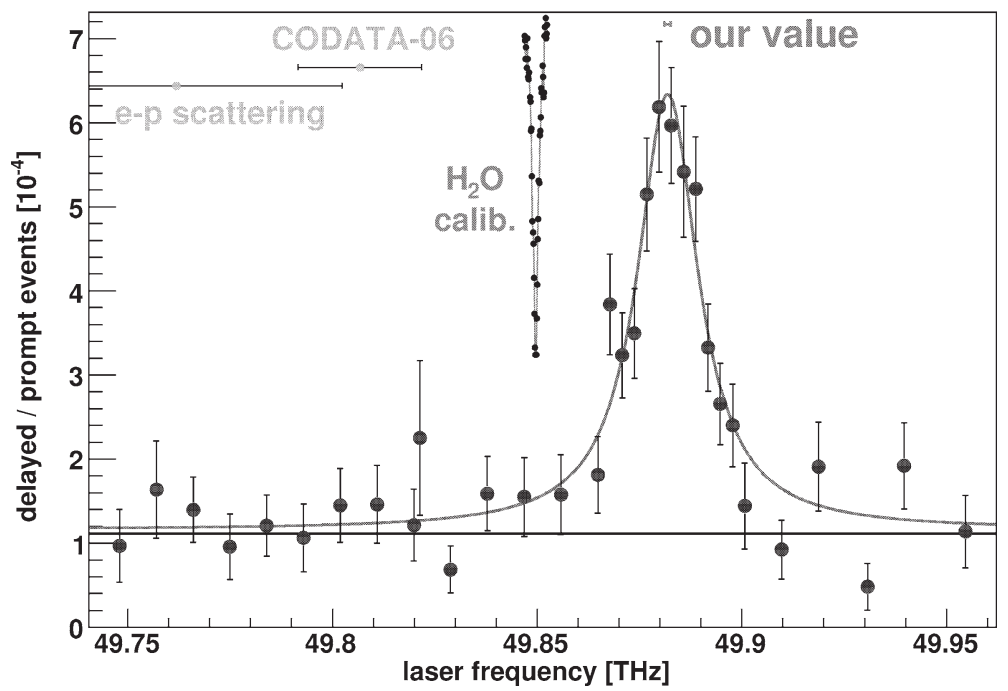

Fig. 5 The resonance as obtained from our data analysis. The $2 \mathrm{~S}_{1 / 2}^{F=1}-2 \mathrm{P}_{3 / 2}^{F=2}$ transition in $\mu \mathrm{p}$ is plotted versus the laser frequency (solid circles). The number of events in the LTW is normalized to the number of prompt events. The fit is a Lorentzian profile on top of a flat background. The predictions for the line position using the proton charge radius from CODATA [36] and from electron scattering $[10,34]$ as well as a water absorption line used for laser frequency calibration are indicated

Our new value for the proton charge radius $r_{\mathrm{p}}=0.84184$ (67) $\mathrm{fm}$ is 10 times more precise, but $5.0 \sigma$ smaller than the currently accepted CODATA-value [36], a discrepancy still subject to vivid discussions in the community. A treatment of possible solutions to this puzzle can be found in [35]. Assuming the theory for the Lamb shift in $\mu p$ [37-42] and for the $\mathrm{H}$ atom [37, 43] to be correct, and using the precisely measured $1 \mathrm{~S}-2 \mathrm{~S}$ interval in $\mathrm{H}[6,8]$, the $1 \mathrm{~S}$ - and $2 \mathrm{~S}$-Lamb shifts in $\mathrm{H}$ calculated with our $r_{\mathrm{p}}$ value, and the most recent value for the fine structure constant $\alpha$ [44], we obtain a new value for the Rydberg constant $R_{\infty}=$ $10,973,731.568160(16) \mathrm{m}^{-1}$ (relative accuracy $1.5 \times 10^{12}$ ). This result is 4.6 times more precise than the CODATA-value [36], but $-110 \mathrm{kHz} / \mathrm{c}$ or $4.9 \sigma$ away.

Physics of simple atomic systems continues to reveal unhidden aspects of Nature's laws. Solutions to the proton size puzzle are expected to come from new investigations in $\mathrm{H}$ (for instance the $2 \mathrm{~S}-4 \mathrm{P}$ transition) and from progress in the $\mu \mathrm{p}$-theory.

\section{References}

1. Heisenberg, W.: Z. Phys. A 33(1), 879 (1925)

2. Dirac, P.: Proc. R. Soc. Lond., A 117(778), 610 (1928)

3. Lamb, W.E., Retherford, R.C.: Phys. Rev. 72(3), 241 (1947)

4. Liu, W., Boshier, M.G., Dhawan, S., van Dyck, O., Egan, P., Fei, X., Grosse Perdekamp, M., Hughes, V.W., Janousch, M., Jungmann, K., Kawall, D., Mariam, F.G., Pillai, C., Prigl, R., zu Putlitz, G., Reinhard, I., Schwarz, W., Thompson, P.A., Woodle, K.A.: Phys. Rev. Lett. 82(4), $711(1999)$

5. Ritter, M.W., Egan, P.O., Hughes, V.W., Woodle, K.A.: Phys. Rev. A 30(3), 1331 (1984) 
6. Parthey, C.G., Matveev, A., Alnis, J., Bernhardt, B., Beyer, A., Holzwarth, R., Maistrou, A., Pohl, R., Predehl, K., Udem, Th., Wilken, T., Kolachevsky, N., Abgrall, M., Rovera, D., Salomon, C., Laurent, P., Hänsch, T.W.: arXiv:1107.3101v1 [physics.atom-ph] (2011)

7. de Beauvoir, B., Schwob, C., Acef, O., Jozefowski, L., Hilico, L., Nez, F., Julien, L., Clairon, A., Biraben, F.: Eur. Phys. J. D 12, 61 (2000)

8. Fischer, M., Kolachevsky, N., Zimmermann, M., Holzwarth, R., Udem, T., Hänsch, T.W., Abgrall, M., Grünert, J., Maksimovic, I., Bize, S., Marion, H., Pereira Dos Santos, F., Lemonde, P., Santarelli, G., Laurent, P., Clairon, A., Salomon, C., Haas, M., Jentschura, U.D., Keitel, C.H.: Phys. Rev. Lett. 92(23), 230802 (2004)

9. Bernauer, J.C., Achenbach, P., Ayerbe Gayoso, C., Böhm, R., Bosnar, D., Debenjak, L., Distler, M.O., Doria, L., Esser, A., Fonvieille, H., Friedrich, J.M., Friedrich, J., Gómez Rodríguez de la Paz, M., Makek, M., Merkel, H., Middleton, D.G., Müller, U., Nungesser, L., Pochodzalla, J., Potokar, M., Sánchez Majos, S., Schlimme, B.S., Širca, S., Walcher, T., Weinriefer, M. Phys. Rev. Lett. 105(24), 242001 (2010)

10. Sick, I.: Phys. Lett. B 576(1-2), 62 (2003)

11. Kusch, P., Foley, H.M.: Phys. Rev. 74(3), 250 (1948)

12. Kottmann, F.: Private communication (2010)

13. Di Giacomo, A.: Nucl. Phys. B 11(2), 411 (1969)

14. Pohl, R.: Investigations of the long-lived metastable $2 S$ state in muonic hydrogen. Ph.D. thesis, ETH Zürich (2001). http://e-collection.ethbib.ethz.ch/view/eth:23936. Accessed June 2011

15. Pohl, R., Daniel, H., Hartmann, F.J., Hauser, P., Kottmann, F., Markushin, V.E., Muhlbauer, M., Petitjean, C., Schott, W., Taqqu, D., Wojciechowski-Grosshauser, P.: Phys. Rev. Lett. 97(19), $193402(2006)$

16. Nebel, T., Amaro, F.D., Antognini, A., Biraben, F., Cardoso, J.M.R., Conde, C.A.N., Dax, A., Dhawan, S., Fernandes, L.M.P., Giesen, A., Hänsch, T.W., Indelicato, P., Julien, L., Knowles, P.E., Kottmann, F., Le Bigot, E., Liu, Y.W., Lopes, J.A.M., Ludhova, L., Monteiro, C.M.B., Mulhauser, F., Nez, F., Pohl, R., Rabinowitz, P., J.M.P. dos Santos, Schaller, L.A., Schuhmann, K., Schwob, C., Taqqu, D., J.Veloso, F.C.A.: Can. J. Phys. 85(5), 469 (2007)

17. Muon and Pion Beams at Paul Scherrer Institute: http://1tp.web.psi.ch/accelerator_and_beams/ pion_muon_beams.htm. Accessed June 2011

18. Jensen, T.S., Markushin, V.E.: Eur. Phys. J. D 21, 261 (2002)

19. Fermi, E., Teller, E., Weisskopf, V.: Phys. Rev. 71(5), 314 (1947)

20. Leon, M., Bethe, H.A.: Phys. Rev. 127(2), 636 (1962)

21. Markushin, V.E.: Phys. Rev. A 50(2), 1137 (1994)

22. Ludhova, L., Amaro, F.D., Antognini, A., Biraben, F., Cardoso, J.M.R., Conde, C.A.N., Dax, A. Dhawan, S., Fernandes, L.M.P., Hänsch, T.W., Hughes, V.W., Indelicato, P., Julien, L., Knowles, P.E., Kottmann, F., Liu, Y.W., Lopes, J.A.M., Monteiro, C.M.B., Mulhauser, F., Nez, F., Pohl, R., Rabinowitz, P., dos Santos, J., Schaller, L.A., Schwob, C., Taqqu, D., Veloso, J.F.C.A., Phys. Rev. A 75(4), 040501 (2007)

23. Anderhub, H., Hofer, H., Kottmann, F., LeCoultre, P., Makowiecki, D., Pitzurra, O., Sapp, B. Seiler, P.G., Wälchli, M., Taqqu, D., Truttmann, P., Zehnder, A., Tschalär, C.: Phys. Lett. B 71(2), 443 (1977)

24. Anderhub, H., von Arb, H.P., Böcklin, J., Dittus, F., Ferreira Marques, R., Hoper, H., Kottmann, F., Taqqu, D., Unternährer, J.: Phys. Lett. B 143(1-3), 65 (1984)

25. Egan, P.O., Dhawan, S., Hughes, V.W., Lu, D.C., Mariam, F.G., Souder, P.A., Vetter, J., G.z. Putlitz, Thompson, P.A., Denison, A.B.: Phys. Rev. A 23(3), 1152 (1981)

26. Pohl, R., Daniel, H., Hartmann, F.J., Hauser, P., Liu, Y.W., Kottmann, F., Maierl, C., Markushin, V.E., Mühlbauer, M., Petitjean, C., Schott, W., Taqqu, D.: Hyperfine. Interact. 138(1-4), 35 (2001)

27. Kottmann, F., et al.: In: Kienle, P., Marton, J., Zmeskal, J. (eds.) Proceedings of the International Workshop on Exotic Atoms (EXA02), p. 159. Austrian Academy of Sciences, Vienna (2003)

28. Simons, L.: Phys. Scr. T22, 90 (1988)

29. Simons, L.: Phys. Bl. 48, 261 (1992)

30. DeCecco, P., Hauser, P., Horváth, D., Kottmann, F., Simons, L.M., Taqqu, D.: Nucl. Instrum. Methods A 394(3), 287 (1997)

31. Antognini, A., Amaro, F., Biraben, F., Cardoso, J., Conde, C., Covita, D., Dax, A., Dhawan, S., Fernandes, L., Hänsch, T., Hughes, V., Huot, O., Indelicato, P., Julien, L., Knowles, P., Kottmann, F., Liu, Y.W., Lopes, J., Ludhova, L., Monteiro, C., Mulhauser, F., Nez, F., Perry, B., Pohl, R., Rabinowitz, P., dos Santos, J., Schaller, L., Schwob, C., Taqqu, D., Veloso, J.: Opt. Commun. 253(4-6), 362 (2005) 
32. Antognini, A., Schuhmann, K., Amaro, F., Biraben, F., Dax, A., Giesen, A., Graf, T., Hänsch, T., Indelicato, P., Julien, L., Cheng-Yang, K., Knowles, P., Kottmann, F., Le Bigot, E., Liu, Y.-W., Ludhova, L., Moschüring, N., Mulhauser, F., Nebel, T., Nez, F., Rabinowitz, P., Schwob, C., Taqqu, D., Pohl, R.: IEEE J. Quantum Electron. 45(8), 993 (2009)

33. Rabinowitz, P., Perry, B., Levinos, N.: IEEE J. Quantum Electron. 22(6), 797 (1986)

34. Blunden, P.G., Sick, I.: Phys. Rev. C 72(5), 057601 (2005)

35. Pohl, R., Antognini, A., Nez, F., Amaro, F., Biraben, F., Cardoso, J., Covita, D., Dax, A., Dhawan, S., Fernandes, L., Giesen, A., Graf, T., Hänsch, T., Indelicato, P., Julien, L., Kao, C.Y., Knowles, P., Lopes, J., Le Bigot, E.O., Liu, Y.-W., Ludhova, L., Monteiro, C., Mulhauser, F., Nebel, T., Rabinowitz, P., dos Santos, J., Schaller, L., Schuhmann, K., Schwob, C., Taqqu, D., Veloso, J., Kottmann, F.: Nature 466(7303), 213 (2010)

36. Mohr, P.J., Taylor, B.N., Newell, D.B.: Rev. Mod. Phys. 80(2), 633 (2008)

37. Eides, M.I., Grotch, H., Shelyuto, V.A.: Phys. Rep. 342(2-3), 63 (2001)

38. Pachucki, K.: Phys. Rev. A 53(4), 2092 (1996)

39. Pachucki, K.: Phys. Rev. A 60(5), 3593 (1999)

40. Borie, E.: Phys. Rev. A 71(3), 032508 (2005)

41. Martynenko, A.P.: Phys. Rev. A 71(2), 022506 (2005)

42. Martynenko, A.P.: Phys. At. Nucl. 71(1), 125 (2008)

43. Karshenboim, S.G.: Phys. Rep. 422, 1 (2005). doi:10.1016/j.physrep.2005.08.008. http://www. citebase.org/abstract?id=oai:arXiv.org:hep-ph/0509010

44. Hanneke, D., Fogwell, S., Gabrielse, G.: Phys. Rev. Lett. 100(12), 120801 (2008) 